\title{
On Standard Model, Topological Skyrme Model and Revival of the Sakaton
}

\author{
Syed Afsar Abbas \\ Centre for Theoretical Physics, JMI University, New Delhi - 110025 \\ Email: drafsarabbas@gmail.com
}

\begin{abstract}
We study the basic conflict between the standard model of particle physics and an another similarly structured but quite different model having the electric charge with color degrees of freedom, and how they connect to a consistent study of QCD for arbitrary number of colors. Further, with the imposition of the consistency with electric charges (i.e. with QED), the topological Skyrme model is shown to lead to a clear revival of the concept of the Sakaton as a physical reality which provides a new perspective to the hypernuclei.
\end{abstract}

Keywords: $\quad$ Standard model, Skyrme model, QCD.

\section{Electric charge in the Standard Model}

The Gell-Mann-Nishijima relation for the "strong" group SU(2) x U(1) was suggested in 1953 as, $\mathrm{Q}=\mathrm{T}_{3}+\mathrm{Y} / 2$ (hypercharge $\mathrm{Y}=\mathrm{B}+$ $\mathrm{S}$, is generator of the $\mathrm{U}(1)$ group). The three quarks as fundamental representation of $\mathrm{SU}(3)$ came much later to Gell-Mannand independently to Zweig in 1964 [1]. In SU(3), $\mathrm{T}_{3}$ and $\mathrm{Y}$ are related as being generators of it. Thus in quark model electric charge is properly quantized. However, Glashow in studying the weak interaction in 1961, incorporated electric charge in a larger electro-weak group $\mathrm{SU}(2)_{\mathrm{W}} \mathrm{x} \mathrm{U}(1)_{\mathrm{w}}$, where the subscript $\mathrm{W}$ refers a different groups defining the Weak and Electromagnetic interactions, in a partially unified manner. Glashow in 1961, just copied the Gell-MannNishijima definition for his electro-weak (EW) group electric charge as $\mathrm{Q}=\mathrm{T}_{3}{ }^{\mathrm{W}}+\mathrm{Y}_{\mathrm{W}}$ 12 . Here $\mathrm{Y}_{\mathrm{W}}$, the weak-hypercharge, is put in by hand. Hence electro-weak model electric charge is not quantized. Glashow in 1961, had no idea of Spontaneous Symmetry Breaking (SSB) by the Englert-Brout-Higgs (EBH) mechanism, which came much later in 1964, and in the EW group in 1969 (Salam and Weinberg). Now the Standard Model (SM), including the strong interaction, extends the EW group structure to $\mathrm{SU}(3) \mathrm{c} \times \mathrm{SU}(2)_{\mathrm{L}} \times$ $\mathrm{U}(1)_{\mathrm{YW}}$. SM is the most successful model of particle physics at present. However the above definitions of the electric charge is carried over in to the SM. Thus electric charge is not quantized and is arbitrary in the SM. This is consided a major weakness of the Standard Model [1]. Note that this unquantized charge in the Standard Model: (1). Prior to any SSB through the EBH field, it already exists in the early universe through some unknown mechanism (2). It is immune or independent of the strong-colour group $\mathrm{SU}(3) \mathrm{c}$; (3). It is fixed with rigid values $2 / 3$ and $-1 / 3$, i.e. no colour dependence; (4). Anomalies play no role other than being trivially satisfied by the above pre-fixed values of the hypercharge in the SM. Quantized Charge Standard Model (QCSM): Hence we have to go beyond the above SM to get quantized charges. We take the same 
generation structure as that in the SM and the same EBH field as an SU(2) $)_{L}$ group doublet, $\left(\begin{array}{l}\phi^{+} \\ \phi^{0}\end{array}\right)$. However, major differences with respect to the above SM are:

(1) We start with the complete group structure as $S U\left(N_{c}\right) \times S U(2)_{L} \times U(1)_{Y_{W}}$ where $\mathrm{N}_{\mathrm{c}}=3$;

(2) We do not have any arbitrarily predefined electric charge;

(3) We take the most general definition of the electric charge in terms of generators of the above group structure as $Q=T_{3}+b Y$ where both $\mathrm{b}$ and $\mathrm{Y}$ are unknown [2].

The first generation fermions are assigned to the following representations

For $S U\left(N_{c}\right) \times S U(2)_{L} \times U(1)_{Y_{W}} ; q_{L}=$ $\left(\begin{array}{l}u \\ d\end{array}\right)_{L},\left(N_{c}, 2, Y_{q}\right) ; u_{R^{\prime}}\left(N_{c}, 1, Y_{u}\right) ; d_{R^{\prime}}\left(N_{o}, 1, Y_{d}\right)$; $l_{R}=\left(\begin{array}{c}v_{e} \\ e\end{array}\right),\left(1,2, Y_{l}\right) ; e_{R},\left(1,1, Y_{\theta}\right)$.

Five unknown hypercharges above plus the unknown $Y_{\phi}$ for EBH field (six unknown to start with). Let the $T_{3}=-\frac{1}{2}$ of the EBH field develop a nonzero vacuum expectation value $\langle\phi\rangle_{0}$. To ensure that one of the four generators $\left(W_{1} W_{2} W_{3}, X\right)$ is thereby left unbroken (meaning that we ensure a massless photon as a generator of the $\mathrm{U}(1)_{\mathrm{em}}$ group), we demand: $Q<\phi>_{0}=0$. This fixes the unknown $b$ and the electric charge now is: $Q=T_{3}+\left(\frac{1}{2 Y_{\phi}}\right) Y$.

For theory to be renormalisable, one ensures that all the anomalies neutralise each other for all the particles known. For each generation, cancellation of anomalies brings in the requirement of three constraints between hypercharges. Before SSB the matter particles are massless. These become massive through this process of SBB by Yukawa couplings, which due to gauge invariance yields three more relations between these unknown hypercharges. These ultimately give relations like $Y_{u}=Y_{\phi}\left(\frac{1}{N_{c}}+1\right)$ and one obtains properly quantized electriccharges in this Quantized Charge Standard Model (which actually thus is a unified model) as [2],

$Q(u)=\frac{1}{2}\left(\frac{1}{N_{c}}+1\right) ; Q(d)=\left(\frac{1}{N_{c}}-1\right) ; Q\left(v_{e}\right)=0 ; Q(e)=-1$

Note that though $\mathrm{U}(1)_{\mathrm{em}}$ does not know of colour, the electric charges are actually dependent upon colour itself. Thus quark charge having colour dependence built into itself, is a significant new result of the Quantized Charge Standard Model. However this is in direct conflict with the Standard Model charges $Q(u)=\frac{2}{3}$ and $Q(d)=-\frac{1}{3}$ (i.e. independent of colour). Which is correct?

\section{QCD in Large Colour limit}

The number of quarks and gluons in $\mathrm{SU}\left(\mathrm{N}_{\mathrm{C}}\right)$ scale as $\sim N_{C} \sim\left(N_{C}^{2}-1\right)$ respectively. So for large $N_{C}$, gluons dominate over quarks. The field theory of $\mathrm{SU}\left(\mathrm{N}_{\mathrm{c}}\right)$ for large $\mathrm{N}_{\mathrm{c}}$ reduces to a theory of weekly interacting mesons, similar to the Skyrme model where baryons arise as topological structures in a Lagrangian composed of scalar mesons only. In this QCD, baryon has a finite size and has a mass going as: $M$ (baryon) $\sim N_{c}$.

Baryons are composed of $\mathrm{N}_{\mathrm{c}}$ number of quarks. Composite baryons to be fermions, $N c$ is an odd number; thus $N_{c}=2 k+1 ; \mathrm{k}=$ $0,1,2 \ldots$ respectively. Now assume that the proton is built up of $(k+1)$ number of $u$ quarks and knumber of $d$-quarks, and viceversa for neutron [2]. Now Witten et.al. took [3] SM quark charges to be independent of colour, $Q_{u}=\frac{2}{3}$ and $Q_{d}=\frac{-1}{3}$ Thus in their model the proton and neutron charges

$Q_{p}=(k+1) \frac{2}{3}+k\left(\frac{-1}{3}\right)=\left(\frac{k+2}{3}\right)=\left(\frac{N_{c}+3}{6}\right)$ 
$Q_{n}=k_{3}^{2}+(k+1)\left(\frac{-1}{3}\right)=\left(\frac{k-1}{3}\right)=\frac{N_{c}-3}{6}$

For arbitrary $\mathrm{N}_{C}$, these are not even integral and actually blow up as $N_{c} \rightarrow \infty$. The colour dependence of proton charge is catastrophic.

Now Witten et. al [3] had unfortunately neglected the fundamental Coulomb selfenergy contribution to the baryon masses. And thus the QCD plus QED contributions to baryon $M($ proton $) \sim N_{c}+C \frac{\left(\frac{N_{c}+\mathrm{s}}{6}\right)^{2}}{R}$, where $C$ is a constant and $\mathrm{R}$ is the finite size of proton. Thus the baryon mass is blowingas $N_{c}^{2}$ due to the QED part. This is messing up the whole analysis based on self-consistent QCD only true for three-colours as well. This is because $M($ proton $) \sim N_{c} \quad$ provides consistent understanding of the relationship between the constituent quarks and the current quarks in QCD for $N_{c}=3$. This $N_{c}^{2}$ dependence is disastrous for the model of Witten et.al.[2]. Thus the definition of electric charge in the Standrd Model is inconsistent with the structure of QCD. Next with our result of colour-dependent electric charges in the Quantized Charge Standard Model,

$Q_{p}=\frac{k+1}{2}\left(1+\frac{1}{N_{c}}\right)+\frac{k}{2}\left(-1+\frac{1}{N_{c}}\right)=1 ; Q_{n}=$ $\frac{k}{2}\left(1+\frac{1}{N_{c}}\right)+\frac{k+1}{2}\left(-1+\frac{1}{N_{c}}\right)=0$

.Thus $Q_{p}=1$ and $Q_{n}=0$ for arbitrary $\mathrm{N}_{\mathrm{c}}$ it is independent of $\mathrm{N}_{\mathrm{c}}$. Hence the Coulomb selfenergy termof the proton remains finite. Thus the colour-dependent electric charge of the QCSM are the proper charges for quarks and proton. Hence electric charges in the QCSM are consistent with QCD while those in the SM are not [2].

3.Topological Skyrme-Wess-Zumino model
The effective action with integer winding number $n \in Z$ and $L_{\mu}=U^{\dagger} \partial U_{\text {; }}$

$U(x)^{s U(3)}=\exp \left(i \frac{\lambda^{a} \phi^{a}(x)}{f_{\pi}}\right) \phi^{a} 0^{-}$meson

octet; Wess-Zumino anomaly (zero for 2 favours) on surface $\Sigma$ is,

$S_{\text {eff }}=\frac{f_{\pi}^{2}}{4} \int d^{4} x\left[L_{\mu} L^{\mu}\right]+n\left(\Gamma_{W 2}=\frac{-i}{240 \pi^{2}} \int d^{5} x \epsilon^{\mu v a \beta} \operatorname{Tr}\left[L_{\mu} L_{v} L_{\alpha} L_{\beta} L_{\gamma}\right]\right)$

On quantizing this theory one obtains a basic quatum number, the right hypercharge $Y_{R}=\frac{1}{3} N_{c} B$, where baryon number $\mathrm{B}$ and $\mathrm{Nc}$ are integers. Note that this right-hypercharge was dictated by having de_ned SU(2) embedding in SU(3). With $B=1$ and $N_{C}=3$ one gets $Y_{R}=1[4,5]$. This identifies the nucleon hypercharge with the body-fixed hypercharge $\mathrm{Y}_{\mathrm{R}}$. Ultimately one obtains a

tower of irreducible representations: $(8,1 / 2)$, $(10,3 / 2),(10,1 / 2),(27,3 / 2), \ldots$ of which the lowest octet and decuplet are identified with the observed low energy baryons. Hence we get all the low dimensional fermions as in the quark model.

This is the standard understanding [4] of the representation of the Skyrme-Wess-Zumino model. However here I show that this is awed, as this was done with incorrect electric charge structure [4,5] of the above octet baryons $(8,1 / 2)$.

Taking $\mathrm{Q}$ as charge operator, under a local electro-magnetic gauge transformation $h(x)=\exp (i \theta(x) Q)$ with small $\theta$, one finds $\Gamma_{W Z} \rightarrow \Gamma_{W Z}-\int d^{4} x \partial_{\mu} J^{\mu}(x)$ where $I_{\mu}$ is the Noether current arising from the WZ term. This coupling to the photon field is,

$I_{\mu}=\frac{1}{48 \pi^{2}} \epsilon^{\mu v \alpha \beta} \operatorname{Tr}\left[Q\left(L_{v} L_{\alpha} L_{\beta}-\right.\right.$

$\left.\left.R_{v} R_{\alpha} R_{\beta}\right)\right]$

where $\quad R_{\mu}=U \partial_{\mu} U^{\dagger}$. With the electromagnetic field $A_{\mu}$ present, the gauge invariant form of effective action 
is, $S_{\text {eff }}=\frac{f_{\pi}^{n}}{4} \int d^{4} x\left[L_{\mu} L^{\mu}\right]+n \Gamma_{W z}$. This means that when replacing the LHS by $\Gamma_{W z}$, then the RHS has two new terms involving $F_{\mu v} F^{\mu v}$. This allows us to interpret $I_{\mu}$ with the current carried by quarks. With the charge operator $\mathrm{Q}, I_{\mu}$ is found to be isoscalar [4]. To obtain the baryon current, one replaces Q by $\frac{1}{N_{C}}$ ( where $N_{C}$ is the number of colours in $\mathrm{SU}\left(\mathrm{N}_{\mathrm{c}}\right)$ QCD for arbitrary number of colours), which is the baryon charge carried by each quark making up the baryon. For total antisymmetry, $\mathrm{N}_{\mathrm{C}}$ number of quarks are needed to make up a baryon. Then $n J_{\mu} \rightarrow J_{\mu}^{B}$ gives,

$J_{\mu}=\frac{1}{48 \pi^{2}}\left(\frac{n}{N_{c}}\right) \epsilon^{\mu v \alpha \beta} \operatorname{Tr}\left[\left(L_{v} L_{\alpha} L_{\beta}-\right.\right.$ $\left.\left.R_{v} R_{\alpha} R_{\beta}\right)\right]=\frac{1}{24 \pi^{2}} \epsilon^{\mu v \alpha \beta} \operatorname{Tr}\left[\left(L_{v} L_{\alpha} L_{\beta}\right)\right]$

This is the same as the topological current of Skyrme. Thus the gauged WZ term gives rise to $I_{\mu}(x)$ which in turn gives the baryon charge. Thus though the WZ term $\Gamma_{W Z}$ is zero for two-flavour case, but $J_{\mu}(x)$ still contibutes there.

What is the meaning of the WZ anomaly term contributing only an isoscalar electric charge? Given a nucleon wave function for $\mathrm{SU}(2), \psi_{N}=\left(\begin{array}{c}\psi_{p} \\ \psi_{n}\end{array}\right)$. Dirac electromagnetic current of proton is $j_{\mu}^{e m}=e \bar{\psi}_{p} \gamma_{\mu} \psi_{p}=e J_{\mu}{ }^{\mathrm{em}}$. The

elecromagnetic current for nucleon is written as

$J_{\mu}{ }^{m m}=\bar{\psi}_{N} \gamma_{\mu} \frac{1}{2}\left(1+\tau_{3}\right) \psi_{N}=J_{\mu}{ }^{m m}(I=0)+$ $I_{\mu}{ }^{\mathrm{m}}(I=1)$.

However we know that in the quark model the electric charge of the baryon octet for each member is given by the Gell-Mann-Nishijima expres-sion $\mathrm{Q}=\mathrm{T}_{3}+\mathrm{Y} / 2$ in its completeness. No breaking into isoscalar and isovector parts.
But here in the Skyrme-Wess-Zumino model, the pure Wess-Zumino anomaly term, to ensure proper interpretation of the baryon number, picks out the pure isoscalar part of the electric charge. So the Wess $\{$ Zumino term contributes pure isoscalar part of the electromagnaetic charge of the nucleon and not separate electric charges of proton and neutron. This is the basic difference of electric charge quantization arising in Skyrme-Wess-Zumino model from that which is there in the quark model for the $(8,1 / 2)$ representation of baryons. So self-consistency demands that the electric charge arising from pure Skyrme Lagrangian be pure isovector. And indeed that is what it is: $Q=\epsilon_{0} L_{3} ; L_{3}$ is $3^{\text {rd }}$ component of isospin operator $\rightarrow Q($ proton $)=\frac{+1}{2}$ and $Q($ neutron $)=\frac{-1}{2}$. Thus charge quantization in Skyrme-Wess-Zumino model is completely different from that of the quark model. So in SWZ model, as one goes to SU(3), the charge quantization should still

be isoscalar -isovector charges. Only particle being $I=0, \quad Y=0$, in $(8,1 / 2)$ octet, allows a pure isoscalar charge for it. Thus one goes from $\left(\begin{array}{l}p \\ n\end{array}\right) \rightarrow\left(\begin{array}{l}p \\ n \\ \Lambda\end{array}\right)$. This is Sakaton of the old SU(3) Sakata model, which was discarded for good reasons. However here the Sakaton is of Skyrmionic nature and says that in hypernuclei only $\Delta$ hyperons should arise. This is true of 42 of 43 hypernuclei experimentally discovered so far. This is a vindication of our revival of the Skyrmionic Sakaton. Hence as per these Skyrmions, this model gives right away the charge of a nucleus for arbitrary number of $\mathrm{Z}$ and $\mathrm{N}$ as, $Q=\frac{z-N}{2}+\frac{z+N}{2}=T_{3}+\frac{A}{2}$.

This well known charge of the nucleus, is obtained here, as nucleus is treated as being made up of Z-protonic skyrmions and $\mathrm{N}$ - 
neutronic skyrmions. Note that we have obtained the fundamental nuclear charge equation directly in terms of the atomic mass number A. Note that this nuclear charge is an anathema for all the presenly successful nuclear physics models.

\section{References:}

1. Abbas, S. A., "Group Theory in Particle, Nuclear, and Hadron Physics", CRC Press, London, 2016.

2. Abbas, S. A., Phys. Lett. 238 (1990) 344;

J. Phys. G 16 (1990) L163.

3. Adkins, G.R., Nappi, C. R. and Witten, E., Nucl. Phys.B 228 (1983) 552.

4. Balachandran, A.P., Marmo, G., Skagerstam, B .A. and A. Stern, "Classical Topology and Quantum States", World Scientific, 1991.

5.Abbas, S. A., Phys. Lett. B 503 (2001) 81. 